\title{
Systematizing Implicit Regularization for Multi-Loop Feynman Diagrams
}

\section{A. L. Cherchiglia*}

Universidade Federal de Minas Gerais

E-mail: alcherchiglia.fis@gmail.com

\section{Marcos Sampaio}

Universidade Federal de Minas Gerais

E-mail: msampaioefisica.ufmg.br

\section{C. Nemes}

Universidade Federal de Minas Gerais

E-mail: carolinaefisica.ufmg.br

\begin{abstract}
Implicit Regularization (IR) is a candidate to become an invariant framework in momentum space to perform Feynman diagram calculations to arbitrary loop order. In this work we present a sytematic implementation of our method that automaticaly displays the terms to be subtracted by Bogoliubov's recursion formula. Therefore, we achieve a twofold objective: we show that the IR program respects unitarity, locality and Lorentz invariance and we show that our method is consistent since we are able to display the divergent content of a multi-loop amplitude in a well defined set of basic divergent integrals in one loop momentum only which is the essence of IR. Moreover, we conjecture that momentum routing invariance in the loops, which has been shown to be connected with gauge symmetry, is a fundamental symmetry of any Feynman diagram in a renormalizable quantum field theory.
\end{abstract}

4th International Conference on Fundamental Interactions August 1-7, 2010

Viçosa, Brazil

\footnotetext{
*Speaker.
} 


\section{Introduction}

A consistent renormalization program in QFT appeared after the work of Bogoliubov, Parasiuk, Hepp and Zimmerman (BPHZ) [1] in which a prescription to extract recursively the divergences of a multi-loop Feynman graph complying with unitarity, locality and Lorentz invariance was presented. The BPHZ program generalized the Dyson's subtraction to general overlapping diagrams leading to the concept of renormalizable quantum field theory. Such program systematizes, according to the topology of the graph, the subtraction necessary to render the corresponding amplitude finite through the forest formula. The proof of finitude provided by the latter is by construction regularization independent. However, for concrete predictions such as scattering amplitudes in collision processes of elementary particles, the method of Dimensional Regularization (DR) and minimal subtraction [2], [3] combined with Zimmerman's forest formula has proven to be an efficient and successful calculational tool particularly for gauge theories. The forest formula can be casted into a counterterm language by means of Bogoliubov's recursion formula [4], complying with locality, Lorentz invariance, unitarity and causality.

To calculate S-matrix elements in a symmetry preserving fashion in a quantum field theoretical model sensitive to dimensional continuation on the space-time, the problem is more subtle. The construction of an invariant regularization framework is aesthetically more appealing but this is not the main motivation. Although in one hand imposing constraint equations derived from Ward identities order by order in perturbation theory obliterates the need of an invariant regularization, on the other hand it renders the calculation more involved from the calculational viewpoint. Besides, if quantum symmetry breakings occur in perturbation theory, an invariant scheme is essential to judge it as physical or spurious. Supersymmetric gauge theories are conspicious examples of models in which regularization and renormalization play a fundamental role especially as new accurate experimental evidence, viz. electroweak precision observables [5], demands consistent theoretical calculations higher than one loop order to understand physics beyond the Standard Model.

Therefore, the construction of an invariant regularization is justified and, in order to be as reliable as DR (wherever DR can be applied), it must be shown to comply with locality, Lorentz invariance, unitarity and causality. Recently, a momentum space framework in the physical dimensional of the quantum field theoretical model (IR) has been developed and shown to be consistent and symmetry preserving in several instances [6]. Thus we believe it to be a promising candidate to become an invariant regularization framework to arbitrary loop order.

The purpose of the present contribution is twofold: first we show how to implement IR in such a way that it displays all the terms to be subtracted by Bogoliubov's recursion formula. Moreover, the local counterterms are left explicit in the form of basic divergent integrals (BDI) in terms of one internal momentum only plus surface terms. The latter are the source of symmetry breaking in all theories IR has been applied to and, therefore, they are always set to zero defining a constrained version of IR (CIR). In this contribution we consider a scalar theory and show that if the surface terms are not set to zero, they will contaminate the renormalization group coefficients. Thus, we are forced to adopt CIR which is equivalent to demand momentum routing invariance in the loops. This feature leads us to conjecture that momentum routing invariance is a fundamental symmetry of any Feynman diagram. 


\section{Systematic implementation of Bogoliubov's recursion formula in IR}

As presented in earlier works [6], the essence of IR is to use an identity at the level of the propagators to free the divergences of the external momenta. However, in order to implement IR in a systematic way to an arbitrary $n$-loop Feynman graph we need to rewrite it in such a way that it evinces the divergent behaviour of the amplitude as the internal momenta go to infinity in all possible ways:

$$
\begin{array}{r}
\frac{1}{\left(k-p_{i}\right)^{2}-\mu^{2}}=\sum_{l=0}^{2\left(n_{i}^{(k)}-1\right)}[\underbrace{\left[\sum_{j=0}^{l l / 2\rfloor} \Theta\left(n_{i}^{(k)}+j-l\right)\left(\begin{array}{c}
l-j \\
j
\end{array}\right) \frac{\left(-p_{i}^{2}\right)^{j}\left(2 p_{i} \cdot k\right)^{l-2 j}}{\left(k^{2}-\mu^{2}\right)^{l+1-j}}\right.}_{f_{l}^{\left(k, p_{i}\right)}}+ \\
+\underbrace{\frac{(-1)^{n_{i}^{(k)}}\left(p_{i}^{2}-2 p_{i} \cdot k\right)^{n_{i}^{(k)}}}{\left(k^{2}-\mu^{2}\right)^{n_{i}^{(k)}}\left[\left(k-p_{i}\right)^{2}-\mu^{2}\right]}}_{\bar{f}^{\left(k, p_{i}\right)}}]
\end{array}
$$

The above identity is the keystone of our procedure which, when applied to a given Feynman graph, can be summarized in the algorithm below:

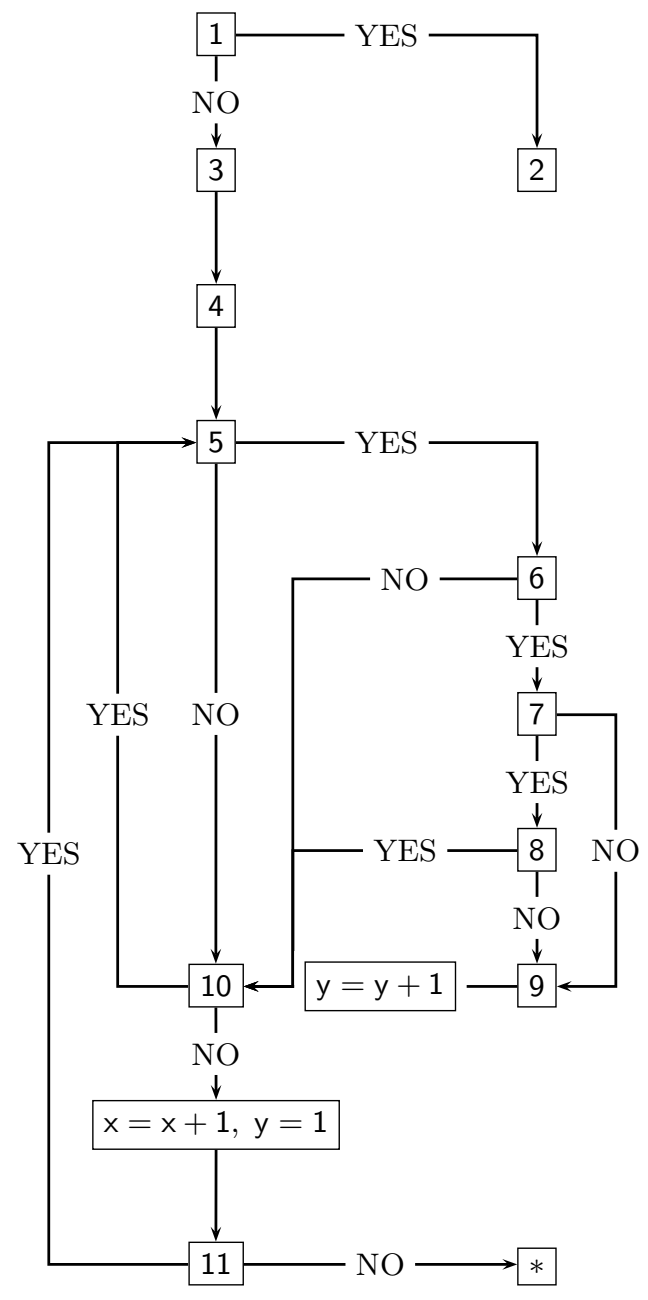


1. Let $\mathscr{S}_{e}$ be the set of subgraphs $G_{i}$ that share one external leg with the whole graph $G$. Let $\overline{\mathscr{S}}_{e}$ be the complementary set. Is $\overline{\mathscr{S}}_{e}$ an empty set?

2. Apply the procedure below (steps $2 \mathrm{a} \rightarrow 2 \mathrm{f}$ ) to the amplitude.

(a) Identify the propagators which depend on the external momenta of the graph and apply identity (2.1);

(b) Find out the minimum value of $n_{j}^{\left(k_{i}\right)}$ needed to assure the finitude of the terms that contain $\bar{f}^{\left(k_{i}, p_{j}\right)}$ as $k_{i} \rightarrow \infty$ in all possible ways;

(c) Repeat the above step for all propagators identified in step 2a;

(d) Identify the divergent terms and classify them according to all possible ways that the internal momenta approach infinity;

(e) Use the rules of IR in the terms identified in step $2 \mathrm{~d}$ according to their classification;

(f) Set aside the divergent terms that contain $I_{\log }^{(l)}\left(\lambda^{2}\right)$ and apply the procedure again on the ones that do not.

3. Identify the propagators which depend on the external momenta and do not belong to a subgraph cointained in $\overline{\mathscr{S}}_{e}$. Use identity (2.1) in such propagators and find out the values of $n_{j}^{\left(k_{i}\right)}$ in the usual way.

4. Set $x=0$ and $y=1$. Group all internal momenta in the set $\overline{\mathscr{K}}_{x=0}^{(y=1)}$ and define its complement (which is an empty set) by $\mathscr{K}_{x=0}^{(y=1)}$.

5. Is there a subgraph $G_{i}$ whose internal momenta are precisely the ones grouped in $\overline{\mathscr{K}}_{x}^{(y)}$ ?

6. Are there divergent terms as all internal momenta contained in $\overline{\mathscr{K}}_{x}^{(y)}$ go to infinity and all elements of $\mathscr{K}_{x}^{(y)}$ are kept fixed?

7. Have all the divergent terms identified in step 6 been stored?

8. Is $\overline{\mathscr{K}}_{x}^{(y)}$ a subset of a already stored $\overline{\mathscr{K}}_{i}^{(j)}(i=0,1, \cdots, x-1)$ ?

9. Store the terms identified in step 6 as well as $\overline{\mathscr{K}}_{x}^{(y)}$ (if $\overline{\mathscr{K}}_{x}^{(y)}$ is a subset of a already stored $\overline{\mathscr{K}}_{i}^{(j)}$, erase the result corresponding to the latter).

10. Choose a new combination of $x$ numbers of internal momenta and group them in the set $\mathscr{K}_{x}^{(y)}$. Is it possible?

11. Choose $x$ numbers of internal momenta and group them in the set $\mathscr{K}_{x}^{(y)}$. Is it possible?

When the algorithm gets to asterisc $(*)$, we write down all the results stored. Each one is classified according to a particular set of internal momenta that go to infinity. If this set has two or more elements, we identify the subgraph that contains them and run the algorithm on it. On the other hand, if the set has only one element we use the rules of IR. At this point, we set aside the terms that contain $I_{\text {log }}^{(l)}\left(\lambda^{2}\right)$ and apply the algorithm on the others. Eventually, we obtain two kind of terms: the ones in which $I_{\log }^{(l)}\left(\lambda^{2}\right)$ multiplies an integral (that correpond to the terms cancelled by 
Bogoliubov's recursion formula) and the ones in which $I_{\log }^{(l)}\left(\lambda^{2}\right)$ multiplies only constants and/or polynominals in the external momenta (that correspond to the typical divergence of the graph).

We applied the method above to the graphs below and in the two-loop ones we kept the surface terms arbitrary. We also computed the coefficients of the renormalization group functions in order to observe if the surface terms play any relevant role in the physics of the underlying quantum field theoretical model. We were particularly interested in the coefficients of the $\beta$-function which are known to be universal.
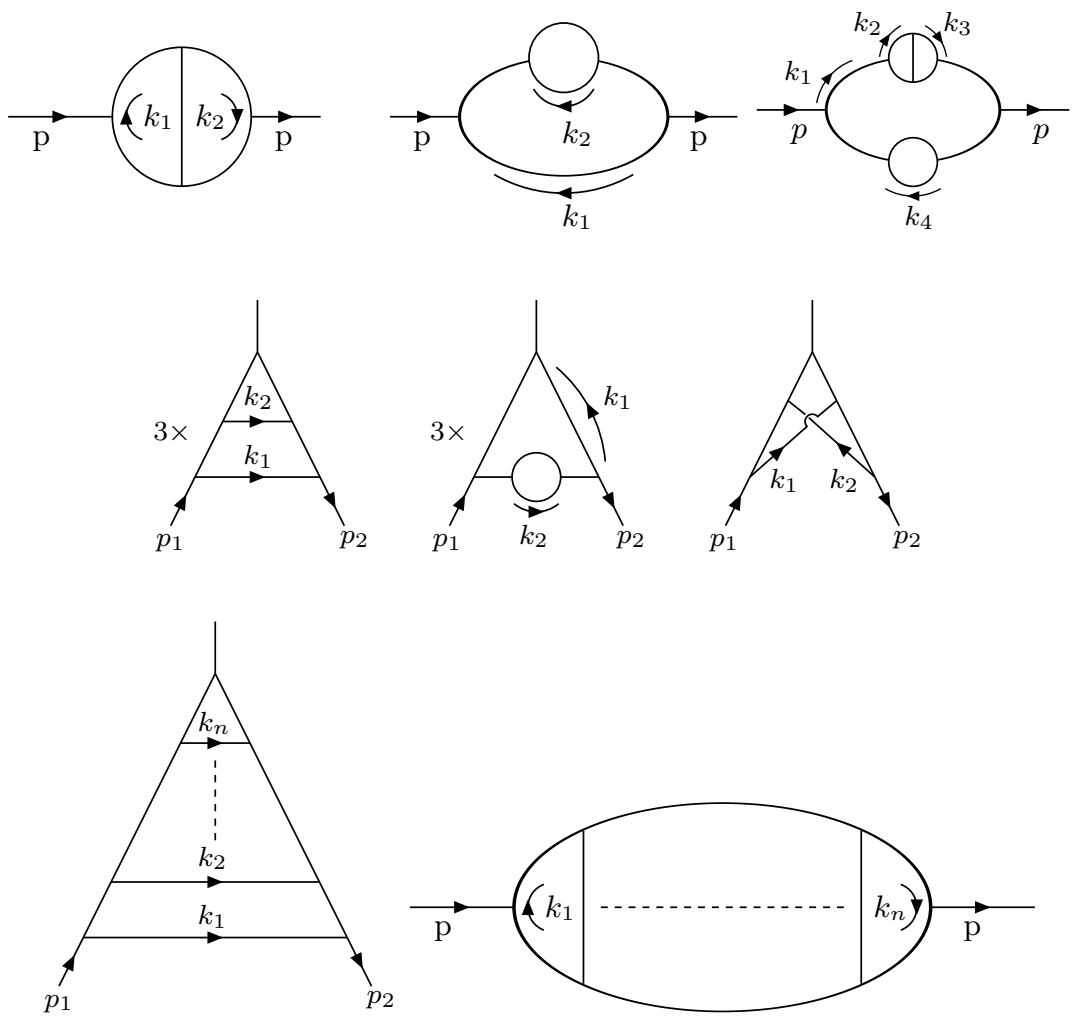

In all the cases above (graphs with nested and/or overlapped subdivergences), our method has found success and the results can be found in [7]. In other words, we were able to express the divergences in terms of BDI's (which is the essence of IR) and the terms to be subtracted by Bogoliubov's recursion formula were automatically displayed (which shows that IR respects unitarity, locality and Lorentz invariance). We also computed the first two coefficients of the $\phi_{6}^{3}$ theory renormalization group functions:

$$
\begin{aligned}
& \gamma=\frac{g^{2}}{12(4 \pi)^{3}}+\frac{13 g^{4}}{432(4 \pi)^{6}}+\frac{i g^{4}}{3(4 \pi)^{3}} \Upsilon_{1}+O\left(g^{6}\right), \\
& \gamma_{m}=\frac{5 g^{2}}{6(4 \pi)^{3}}+\frac{97 g^{4}}{108(4 \pi)^{6}}+\frac{16 i g^{4}}{3(4 \pi)^{3}} \Upsilon_{1}+O\left(g^{6}\right), \\
& \beta=-\frac{3 g^{3}}{4(4 \pi)^{3}}-\frac{125 g^{5}}{144(4 \pi)^{6}}-\frac{5 i g^{5}}{(4 \pi)^{3}} \Upsilon_{1}+O\left(g^{6}\right) .
\end{aligned}
$$


Since the surface terms are arbitrary and appear in the $\beta$-function universal coefficients, they must be set to zero which corresponds to invoking momentum routing invariance in the loops. Therefore, we are lead to conjecture that this is a fundamental symmetry of Feynman graphs.

\section{Concluding Remarks}

We developed a systematic implementation of Implicit Regularization that complies with Bogoliubov's recursion formula and thus we have shown that IR respects unitarity, locality and Lorentz invariance. Moreover, we found that momentum routing invariance (which is connected to gauge symmetry) is a must even for theories with less symmetry content (like scalar ones). This feature leads us to conjecture that it is a fundamental symmetry of any Feynman diagram in a renormalizable quantum field theory.

\section{References}

[1] N. N. Bogoliubov and O. S. Parasiuk, Acta Math. 97 (1957) 227; O. S. Parasiuk, Ukrain. Mat. Zh. 12 (1960), 287; N. N. Bogoliubov and O. V. Shirkov, "Introduction to the Theory of Quantized Fields", 4th ed., Wiley, New York (1980); K. Hepp, Commun. Math. Phys. 2 (1966) 301; "La Theorie de la Renormalisation", Lect. Notes in Physics 2 Springer (1969); W. Zimmermann, Commun. Math. Phys. 11 (1968) 1; Commun. Math. Phys. 15 (1969) 208.

[2] C. G. Bollini and J. J. Giambiagi, Nuovo Cimento B 12 (1972) 20.

[3] G. 't Hooft and M. J. G. Veltman, Nucl. Phys. B 44 (1972) 189.

[4] T. Muta, "Foundations of QCD", World Scientific, Singapore (1987).

[5] S. Heinemeyer, W. Hollik, D. Stockinger, A. M. Weber and G. Weiglein, J. High Energy Phys. 0608 (2006) 052; J. R. Ellis, S. Heinemeyer, K. A. Olive, A. M. Weber and G. Weiglein, J. High Energy Phys. 0708 (2007) 083; S. Heinemeyer, X. Miao, S. Su and G. Weiglein, J. High Energy Phys. 0808 (2008) 087; A. Falkowski and M. Perez-Victoria, J. High Energy Phys. 0912 (2009) 061.

[6] O. A. Battistel, A. L. Mota and M. C. Nemes, Mod. Phys. Lett. A 13 (1998) 1597; A. P. Baeta Scarpelli, M. Sampaio, B. Hiller and M. C. Nemes, Phys. Rev. D 64 (2001) 046013; A. P. Baeta Scarpelli, M. Sampaio and M. C. Nemes, Phys. Rev. D 63 (2001) 046004; M. Sampaio, A. P. Baeta Scarpelli, B. Hiller, A. Brizola, M. C. Nemes and S. Gobira, Phys. Rev. D 65 (2002) 125023; M. D. Sampaio, A. P. Baeta Scarpelli, J. E. Ottoni and M. C. Nemes, Int. J. Theor. Phys. 45 (2006) 436; C. R. Pontes, A. P. Baeta Scarpelli, M. Sampaio, J. L. Acebal and M. C. Nemes, Eur. Phys. J. C 53 (2008) 121; O. A. Battistel and M. C. Nemes, Phys. Rev. D 59 (1999) 055010; O. A. Battistel, PhD thesis, Federal University of Minas Gerais (2000); D. E. Carneiro, A. P. Baeta Scarpelli, M. Sampaio and M. C. Nemes, J. High Energy Phys. 0312 (2003) 044; L. A.M. Souza, M. Sampaio and M. C. Nemes, Phys. Lett. B 632 (2006) 717; J. E. Ottoni, A. P. Baeta Scarpelli, M. Sampaio and M. C. Nemes, Phys. Lett. B 642 (2006) 253; E.W. Dias, B. Hiller, A. L. Mota, M. C. Nemes, M. Sampaio and A. A. Osipov, Mod. Phys. Lett. A 21 (2006) 339; B. Hiller, A. L. Mota, M. C. Nemes, A. A. Osipov and M. Sampaio, Nucl. Phys. A 769 (2006) 53; O. A. Battistel and G. Dallabona, Eur. Phys. J. C 45 (2006) 721; C. R. Pontes, M. C. Nemes, A. P. Baeta Scarpelli and M. Sampaio, J. Phys. G 34, 2215 (2007); A. P. B. Scarpelli, M. Sampaio, M. C. Nemes and B. Hiller, Eur. Phys. J. C 56 (2008) 571; E. W. Dias, A. P. Baeta Scarpelli, L. C. T. Brito, M. Sampaio and M. C. Nemes, Eur. Phys. J. C 55 (2008) 667.

[7] A. L. Cherchiglia, M. Sampaio, M. C. Nemes, [hep-th/1008.1377] 